PIXEL 2008 INTERNATIONAL WORKSHOP

FERMILAB, BATAVIA, IL, U.S.A.

23-26 SEPTEMBER 2008

\title{
The control systems of the CMS Pixel detector
}

\section{Christian Veelken}

Department of Physics, University of California, One Shields Avenue, Davis, CA 95616, U.S.A.

E-mail: christian.veelken@cern.ch

ABSTRACT: The CMS pixel detector measures the direction and momentum of charged particles close to the nominal interaction point of the CMS experiment at the CERN Large Hadron Collider (LHC). The pixel detector is a rather complex device, consisting of 1444 detector modules, in total providing about 66 million pixels of size $100 \times 150 \mu \mathrm{m}$ and an active area of about $1 \mathrm{~m}^{2}$ for the measurement of charged particles traversing its fiducial volume. The control systems of the CMS pixel detector will be described in this document.

KEYWORDS: Particle tracking detectors; Detector control systems (detector and experiment monitoring and slow-control systems, architecture, hardware, algorithms, databases) 


\section{Contents}

1 Introduction 1

2 Detector Control System 2

2.1 CAEN EASY power supply system 3

2.2 Siemens S7-300 PLC system 4

2.3 PVSS 6

\section{Introduction}

Silicon pixel detectors have been installed in the CMS experiment at the CERN Large Hadron Collider (LHC) for b-jet and $\tau$ tagging purposes as well as for the purpose of seeding the track reconstruction algorithm in the silicon strip tracker surrounding the pixel detector. The pixel detector is divided into a barrel and an endcap part. The barrel part consists of three concentric shells of length $570 \mathrm{~mm}$, located at radii of $44 \mathrm{~mm}, 73 \mathrm{~mm}$ and $102 \mathrm{~mm}$. The endcap part consists of a total of 4 pairs of half-disks (with each pair mated to form a full disk), 2 pairs on either end of the the barrel. ${ }^{1}$ The disks are located at $z= \pm 345 \mathrm{~mm}$ and $z= \pm 465 \mathrm{~mm}$. They extend from about $60 \mathrm{~mm}$ to $150 \mathrm{~mm}$ in radius. The combination of barrel and endcap pixel detectors provides between 2 and 3 space-points for tracks reconstructed within the pseudo-rapidity range $-2.5<\eta<+2.5$ [1].

As a result of being located in the immediate vicinity to the interaction region of the CMS experiment, high radiation doses are anticipated for the pixel detector. Its components have been designed to withstand a particle fluence (principally charged hadrons) of up to $6 \times 10^{14} \mathrm{~cm}^{-2}$ [1]. The dominant fraction of this radiation dose is expected to be due to the particles produced in $p p$ collisions and is hence an unavoidable by-product of normal data-taking. In order to reduce the effects of radiation damage, the pixel detector needs to be kept cold, at a temperature of about $-10^{\circ} \mathrm{C}$, after the first year of (low luminosity) LHC operation. The detector is cooled by liquid $C_{6} F_{14}$. The capacity of the fluoro-carbon cooling system has been designed to cool the detector down to temperatures below $-10^{\circ} \mathrm{C}$ and to keep it at that temperature during data-taking, removing of the order of $4 \mathrm{~kW}$ of heat generated by the electronics components of the pixel detector and its active elements, the pixel sensors.

Two separate systems have been built in order to control the CMS pixel detector. The detector control system (DCS), traditionally referred to also as "slow controls" system, monitors environmental conditions and controls the power supplies. It is complemented by a "fast controls" system of high bandwidth and short response time, the task of which is to control the on-detector electronics.

\footnotetext{
${ }^{1}$ The installation of a third endcap disk on either side of the barrel is foreseen as a possible future upgrade of the CMS pixel detector.
} 


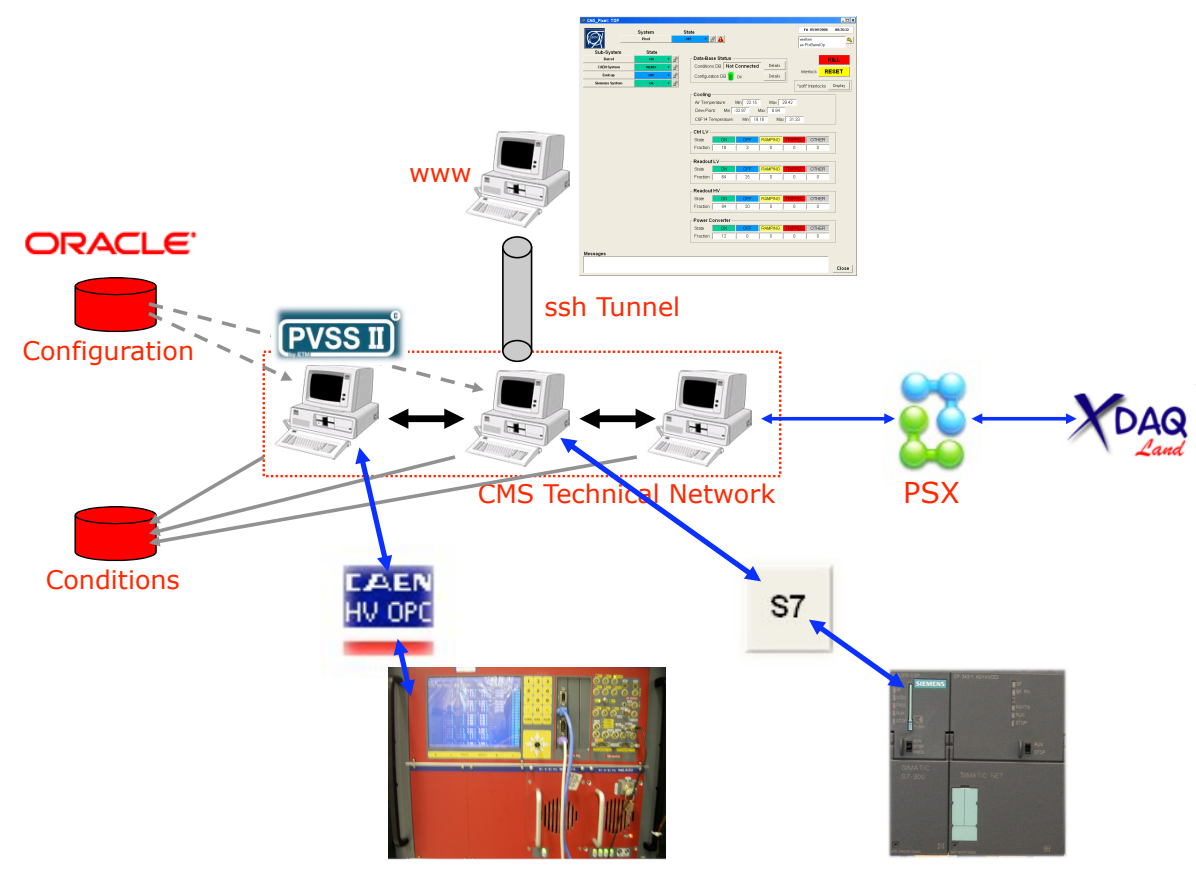

Figure 1. Schematic of the CMS pixel DCS.

In the following, the hardware and software components of the CMS pixel detector control system (DCS) are described. The hardware of the fast controls system has already been described in the last Pixel conference and we would like to refer the reader to the proceedings [2]. At time of writing this document, the documentation of the software components of the fast controls system is in preparation [3].

\section{Detector Control System}

The purpose of the DCS is twofold: first, its task is to control and monitor the high voltage (HV) and low voltage (LV) power supplies providing the bias voltage for the depletion of the pixel sensors and powering the on-detector read-out electronics; its second task is to monitor the temperature and humidity conditions, in order to guarantee that operation of the detector is safe at all times.

The system architecture of the DCS is depicted in figure 1. Its main hardware components, the CAEN power supply system and the Siemens S7-300 programmable logic controller (PLC) system (which performs the monitoring of the temperature and humidity conditions) are symbolized in the bottom of the figure. Both systems are controlled by PVSS $^{2}$ [4], the CERN solution for SCADA $^{3}$ applications. PVSS is connected to Oracle databases to retrieve configuration parameter settings and to archive conditions data - temperature, humidity, voltage and current readings. The PVSS software is installed on rack-mounted Windows XP systems located in the USC55 underground counting room of the CMS experiment. Multiple PVSS graphical user interface processes, executed by other Windows XP or Linux systems, can connect to the underground PVSS systems

\footnotetext{
${ }^{2}$ Prozessvisualisierungs- und Steuerungssystem.

${ }^{3}$ Supervisory Control and Data Acquisition.
} 
simultaneously via standard TCP/IP communication in the CMS technical network. Gateways allow for clients not within the domain of the CMS technical network to connect to the underground PVSS system via ssh tunnels.

An interface (denoted by PSX [5] in figure 1) allows the DCS to exchange information with the fast controls system (denoted by "XDAQ"). The interface implements a hand-shaking protocol for the turn-on sequence of the endcap pixel detector and, in addition, permits one to increase the number of temperature sensors that can be monitored by the DCS beyond the limit that can be read-out via hardwired cables, by forwarding temperature information read-out via the data-stream of the fast controls system to the DCS [3].

\subsection{CAEN EASY power supply system}

The high and low voltages needed for operation of the CMS pixel detector are provided by a modular power supply system of type CAEN EASY ${ }^{4}$ [6]. The main component of the system is a mainframe of type SY1527, located in the USC55 underground counting room. The mainframe hosts an Ethernet interface which allows monitoring and control of the CAEN system via PVSS. The actual power supply boards are mounted in two racks located on the galley of the USX55 experimental hall, about $100 \mathrm{~m}$ away from the USC55 underground counting room. The racks are controlled by the mainframe by means of two branch-controller boards of type A1676, which are plugged-into the mainframe. The separation of the SY1527 mainframe from the actual power supply boards allows the control logic circuits of the mainframe to be located in a low-radiation, low magnetic field area, while the radiation hard and magnetic field resistant power supply boards can be located close to the detector, reducing the voltage drops (and hence power losses) in the power cables.

Two types of boards, custom designed by CAEN, are used in the CMS pixel power supply system. Boards of type A4602 provide 4 low voltage channels of nominal output voltages $2.5 \mathrm{~V}$ which power on-detector electronics components of the "fast controls" system. A4603 type boards contain two electrically independent power supply units (PSUs), mounted within the same mechanical enclosure. Each PSU provides 2 LV plus 2 HV channels. The two LV channels provide nominal output voltages of $1.8 \mathrm{~V}$ and $2.5 \mathrm{~V}$ for the analog and digital circuits of the pixel read-out chips. In order to account for variations in the voltages necessary to deplete sensors after irradiation, two separate HV channels allow lower (higher) bias voltages to be applied to sensors located at a larger (smaller) distance from the beam axis. All boards are equipped with a microprocessor, which allows control and monitoring of voltages and currents, trip-times and ramp-up/ramp-down rates. Voltage regulators allow compensation for current dependent voltage drops in the power cables by continuously measuring via sense wires the voltages at the detector end and adjusting the voltages at the board end of the power cables so as to keep the voltages provided to the detector constant. The properties of LV and HV channels of A4602 and A4603 type boards are summarized in table 1.

In total, 14 boards of type A4602 and 48 boards of type A4603 are providing power to the CMS pixel detector. ${ }^{5}$ The boards are mounted in 10 EASY4000 crates, 5 crates of boards in each

\footnotetext{
${ }^{4}$ Embedded Assembly SYstem.

${ }^{5}$ These values are for the present endcap pixel configuration with two disks installed on either side of the barrel. In case of a possible future upgrade to a three disk configuration, 14 boards of type A4602 and 56 boards of type A4603 are necessary.
} 
Table 1. Channel characteristics of CAEN A4602 and A4603 boards.

\begin{tabular}{|l|c|ccc|}
\hline Channel Type & $\begin{array}{c}\text { A4602 } \\
\text { LV }\end{array}$ & \multicolumn{3}{|c|}{ A4603 } \\
& $\begin{array}{c}\text { digital LV } \\
\text { analog LV }\end{array}$ & HV \\
\hline Voltage at Load (v0) & $2.25-2.75 \mathrm{~V}$ & $1.8-3.0 \mathrm{~V}$ & $1-2.3 \mathrm{~V}$ & $0-600 \mathrm{~V}$ \\
Voltage at Connector (vConn) & $<8 \mathrm{~V}$ & $<5.8 \mathrm{~V}$ & $<7 \mathrm{~V}$ & $0-600 \mathrm{~V}$ \\
Voltage Monitor Resolution & $5 \mathrm{mV}$ & $5 \mathrm{mV}$ & $5 \mathrm{mV}$ & $100 \mathrm{mV}$ \\
\hline Current (i0) & $<7 \mathrm{~A}$ & $<6 \mathrm{~A}$ & $<13 \mathrm{~A}$ & $0-20 \mathrm{~mA}$ \\
Current Monitor Resolution $^{1}$ & $7 \mathrm{~mA}$ & $10 \mathrm{~mA}$ & $13 \mathrm{~mA}$ & $20 \mu \mathrm{A}$ \\
\hline Ramp-up/Ramp-Down Rate $^{2}$ & - & - & - & $1-100 \mathrm{~V} / \mathrm{s}$ \\
\hline Output Power & $<56 \mathrm{~W}$ & $<34.8 \mathrm{~W}$ & $<91 \mathrm{~W}$ & $<12 \mathrm{~W}$ \\
\hline Number of Channels & $48^{3}$ & 96 & 96 & 192 \\
used for CMS pixel detector & & & & \\
\hline
\end{tabular}

\footnotetext{
${ }^{1}$ Note that the resolutions quoted for the current monitor are slightly different from the values specified in the CAEN documentation. The reasons are twofold: first, the currents are digitized by an ADC with 10-bit resolution; the resolution of the current monitor is hence equal to $1 / 2^{10} \mathrm{i} 0$. Second, the precision of the current monitor readings are limited by the number of digits made available for read-out by the firmware of the A4602 and A4603 boards. For the low voltage channels of A4602 type boards, the value of the least significant digit of the current monitor is $10 \mathrm{~mA}$. The low voltage channels of A4603 type boards had the same value for the least significant digit initially; in order to increase the precision of the read-out, the value was subsequently reduced to $1 \mathrm{~mA}$ by CAEN engineers upon request by the CMS pixel detector group.

${ }^{2}$ Configurable in steps of $1 \mathrm{~V} / \mathrm{s}$.

${ }^{3} 8$ channels of the 14 boards of type A4602 are not used.
}

rack, providing power to the $+z(-z)$ side of the detector. The EASY4000 crates are powered by three 3-phase $230 \mathrm{~V}_{A C}$ to $48 \mathrm{~V}_{D C}$ power converters of type $\mathrm{A} 3486 \mathrm{H}$ which are mounted in the same racks, above the 5 crates filled with A4602 and A4603 type boards.

\subsection{Siemens S7-300 PLC system}

In case radiation levels, temperature or humidity values represent a threat for the safe operation of the pixel detector, the low and high voltage channels of the CAEN EASY power supply system get ramped-down and kept switched-off. The exact conditions defining when the detector is threatened by irradiation, overheating or condensation damage are determined by control logic programmed into a commercial Siemens S7-300 PLC system. The PLC system reads-out 80 (120) Pt1000 RTD $^{6}$ temperature sensors plus 4 (4) HMX humidity sensors [7] mounted within the volume of the CMS barrel (endcap) pixel detector and receives radiation warning and error signals from the beam conditions monitor (BCM) [8].

The PLC system is built in a modular design. It consists of 5 crates filled with modules which are mounted in one rack located in the USC55 underground counting room. Each crate is equipped with its own patch-panel and can be installed (or removed for maintenance) separately

\footnotetext{
${ }^{6}$ Resistance Temperature Detector.
} 
from the other crates. The main component of the Siemens S7-300 PLC system is a programmable logic controller module of type CPU319-3 PN/DP, that processes the safety program and hosts an ethernet interface which allows the PLC to communicate with PVSS. The signals of the 8 humidity sensors are read-out by 1 (generic) analog input module. Due to the fact that the signal provided by the HMX sensors is of the order of a few millivolt only, the signals need to get amplified before they can be sampled by the analog input module. The necessary amplification is performed by a conditioning electronics board developed by the CMS silicon strip tracker group. The temperature sensors are read-out by a special type of analog input module, dedicated to the read-out of RTD sensors. In total, 32 of these dedicated RTD modules are used in the CMS pixel PLC system. The warning and error signals provided by the $\mathrm{BCM}$ are read-out via 1 digital input module. In case the safety of the pixel detector is threatened, either by radiation levels, by temperature or by humidity conditions, relays get opened by the control logic programmed into the PLC module (the safety program), to interrupt interlock lines that are connected to the CAEN EASY4000 crates, which causes the power supply boards mounted in the crates to ramp-down and stay switched-off. A total of 4 relay modules is used in the CMS pixel PLC system, which permits the A4602 and A4603 type boards of each barrel (endcap) pixel half-cylinder (half-shell) to be interlocked separately. A Siemens Sitop battery-powered uninterruptible power supply (UPS) is installed in the bottom of the rack, allowing the PLC system to keep processing the safety program in case of power outages.

The safety program has been developed specifically for the CMS pixel detector. It is implemented by means of SCL ("Structured Control Language") [9], a Siemens specific programming language similar to Pascal, and consists of two parts: the handling of configuration parameters downloaded by PVSS into the PLC and the actual interlock control logic which evaluates radiation levels, temperature or humidity conditions and opens the relays interlocking the CAEN power supplies if required in order to protect the pixel detector from potential damage.

The safety program has been designed with the aim of being highly flexible, allowing its behavior to be adjusted to requirements which may be evolving during the lifetime of the CMS experiment. In the chosen design, the behavior of the PLC system can be adjusted to a high degree, by downloading suitable configuration parameters at run-time. In order to prevent the system from behaving in erroneous ways, the configuration parameters downloaded by PVSS are subject to thorough validity and consistency checks before they get used in the control logic part of the safety program.

The control logic part evaluates which temperature sensors are above the limits configured for overheating alarms, computes dew-points from a combination of temperature and humidity readings, evaluates which relative humidity and dew-point values are within the limits configured for condensation alarms and checks the status of radiation warning and error signals received from the BCM. It allows alarms to be delayed by a configurable time, in order to increase the robustness against temporary glitches by suppressing alarms which last only for a short period of time. In case temperature or humidity sensors have been in alarm or the radiation warning/error signals of the BCM have been active for longer then the configured alarm delay, interlocks are triggered by the safety program. A majority logic implemented in the program allows one to specify that $m$ out of a group of $n$ temperature or humidity sensors need to be in alarm condition before an interlock triggers. Finally, relays get opened by the safety program in case interlocks trigger. The relays which get opened can be configured for each interlock independently.

The safety program is executed in an endless loop, with a cycle-time of about once every $50 \mathrm{~ms}$. 


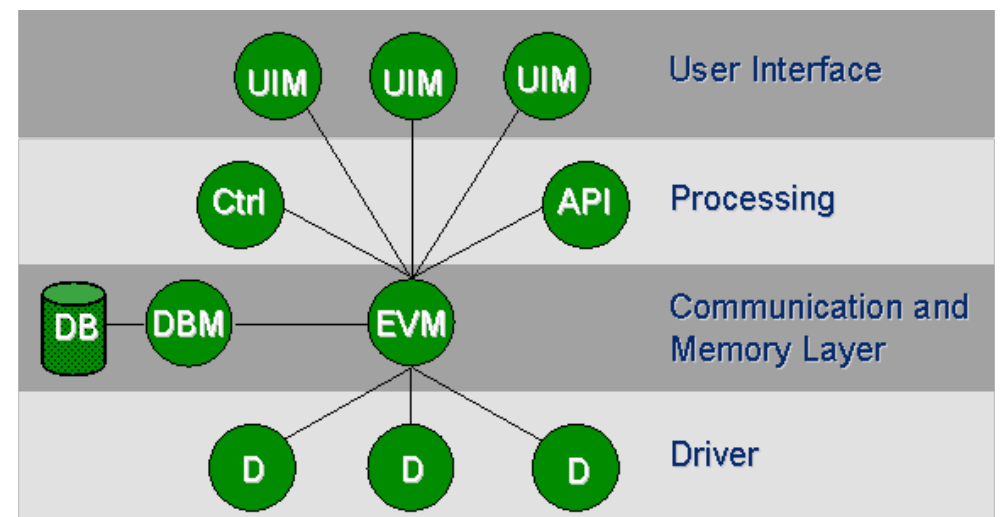

Figure 2. Architecture of PVSS installations. Independent manager processes are depicted by circles, the communication via TCP/IP between different managers is depicted by lines. Principal component of each PVSS installation is the event manager (EVM). Driver (D) processes may handle the low-level communication with different types of hardware. Purpose of the database manager (DBM) is to provide an interface to Oracle databases. Control managers (Ctrl) may execute control scripts implemented in the PVSS scripting language. The architecture supports the connection of multiple (graphical) user interface managers (UIM) to the event manager process.

\subsection{PVSS}

PVSS is the central component of the CMS pixel detector control system. It provides interfaces to the CAEN EASY power supply and Siemens S7-300 PLC systems, interfaces to Oracle databases, a scripting language (similar to C) for control scripts, support for graphical user interfaces and an application programming interface (API), which allows the functionality provided by "standard" PVSS to be extended. Individual components of the PVSS software package are implemented as managers, independent processes which communicate with one another via standard TCP/IP communication. The individual managers allow the load of PVSS installations to be shared in a very transparent way between multiple Windows XP or Linux systems. The general architecture of PVSS installations is illustrated in figure 2.

The PVSS software package includes different interfaces for low-level communication with the hardware. Board and channel parameters of the A4602 and A4603 type CAEN EASY power supply boards are controlled and monitored via the Ethernet interface of the SY1527 mainframe, by means of an $O P C^{7}$ server software provided by CAEN [10]. The OPC server software is executed by one of the rack-mounted Windows XP systems depicted in figure 1. It communicates via standard TCP/IP with a generic OPC client manager process (symbolized by a "D" in figure 2) of PVSS. The status of temperature and humidity sensors, the interlocks and the relays of the Siemens S7-300 PLC system is monitored via the Ethernet interface of the CPU319-3 PN/DP module by means of a PVSS native S7-driver [11].

The data-points (the PVSS equivalent of data-structures) associated with parameters of the CAEN power supply and Siemens PLC systems which are monitored and controlled by PVSS are created by an automatic procedure, by means of mass-configuration scripts. The mass-configuration scripts create the data-points, configure the connections of data-points to the CAEN OPC server

\footnotetext{
${ }^{7}$ Object linking and embedding for Process Controls.
} 
and to the PVSS native S7-driver, configure the archiving options for the PVSS conditions database and initialize the elements of data-points (the individual variables contained in the data-structures) with default values. The information needed by the scripts is taken from Excel spreadsheets ${ }^{8}$ at the moment. In the future it is foreseen that all information needed by the mass-configuration scripts will be stored in an Oracle database. Data-point types specific to the CMS pixel detector have been developed to represent the parameters of the Siemens S7-300 PLC system. Parameters of the CAEN EASY power supply system are represented by data-point types provided by the JCOP framework [12].

After the data-points have been created, configured and initialized by the mass-configuration scripts, they are stored in the PVSS configuration database [13]. The PVSS configuration database tool stores the complete history of all changes to data-point values in Oracle database tables, thereby allowing their evolution to be tracked. A feature which is not used by the CMS pixel DCS right now, but might turn-out to be very useful in the future, is the ability of the PVSS configuration database to switch between different sets of configuration parameters.

In order to control the $\mathscr{O}(500)$ data-points that represent low and high voltage channels of the CAEN A4602 and A4603 power supply boards and the $\mathscr{O}(200)$ data-points that represent temperature and humidity values read-out by the Siemens PLC system, the Finite State Machine (FSM) toolkit [14] of the JCOP framework is used. The FSM toolkit also allows implementation of the turn-on sequences of the barrel and endcap pixel detectors.

In the FSM, the different devices of the CAEN and Siemens systems are organized in a treelike hierarchical structure, modeling the partitioning of the detector into its individual component parts. Physical devices such as LV and HV channels, as well as temperature and humidity sensors are represented by leafs at the bottom of the tree-like hierarchy. The leafs are instances of device units (DUs). At least one device unit type has been defined for each data-point type. ${ }^{9}$ The leafs are connected into a tree-like structure by logical units (LUs) and control units (CUs), further up in the hierarchy. The LUs and CUs are purely logical nodes, which do not represent any hardware. The purpose of the logical nodes is to twofold: to propagate operator commands to the leafs (the physical devices) at the bottom of the hierarchy and to summarize the status of physical devices, in order to reduce at each level of the FSM hierarchy the complexity of detector status information presented to an operator. ${ }^{10}$ The DUs, the LUs and the CUs are defined in terms of a set of states plus a set of transitions that are possible between the states. The transitions can be initiated either manually, by operator commands, or by automatic actions, triggered by DUs, LUs or CUs entering a certain state. By suitable choices of the set of transitions that are possible in each state, manual and automatic sequences for the detector turn-on procedure have been implemented.

\footnotetext{
${ }^{8}$ The method has been developed by the CMS silicon strip tracker DCS group and has been adapted to the specifics of the CMS pixel detector.

${ }^{9}$ In case different behavior is demanded for devices of one and the same data-point type, two or more device unit types can be defined for the same data-point type.

${ }^{10}$ The difference between LUs and CUs is that nodes that are instances of CUs can be operated independently by different operators, whereas instances of LUs that are below the same CU cannot be operated by different operators. The partitioning of the FSM hierarchy into independent domains managed by CUs allows e.g. for an expert to be in control of and debug one part of the pixel detector (which is excluded from normal data-taking), while all the other parts of the pixel detector are under the control of an operator and are included in normal data-taking. In the FSM hierarchy of the CMS pixel detector, the 4 half-shells (4 half-cylinders) of the barrel (endcap) pixel detector, the barrel and endcap parts of the pixel detector and the pixel detector as a whole are represented by independent CUs.
} 
Approximately 100 CMS pixel specific graphical user interface (GUI) panels have been developed for the operation of physical devices of the CAEN and Siemens systems. Three different categories of graphical user interfaces have been implemented: panels summarizing the status of individual component parts of the detector, panels depicting the status of the Siemens system, and panels depicting the status of the CAEN system. The first category of panels has been designed to alert the operator of potential problems, which may either cause damage to the detector or affect the quality of the physics data taken. The operator panels are linked to different levels of the FSM hierarchy. Different degrees of summarization of the detector status information have been implemented to guide the attention of the operator when operating the FSM. Once a potential problem has been spotted by an operator, the second and third categories of GUI panels allow the problem to be investigated by experts in more detail. The second category of panels allow problems related to the Siemens S7-300 PLC system to be investigated in greater detail. Specifically developed panels allow the status of the last configuration parameter download to be checked and detailed hardware status and diagnostics information to be displayed. The third category consists of panels that allow detailed status information for the CAEN system to be displayed. For the latter, panels provided by the JCOP framework [12] are used.

Groups of 5 to 10 operators have been trained to operate the CMS pixel DCS system in training sessions of duration between one and two hours. During the training sessions, a software tool is used which simulates the responses of the Siemens and CAEN systems and that of the pixel detector. The tool allows experience to be gained in operating the FSM without any access to (hence risk of damaging) real hardware. A user's manual introducing operators to the concepts of the CMS pixel DCS and describing its graphical user interfaces is provided.

Releases of the CMS pixel specific PVSS software components are built in regular intervals. ${ }^{11}$ A cvs [15] repository is used to maintain the code, allowing multiple developers to work on the same code at the same time. For a group such as ours, spread between different locations in the United States and at CERN, cvs turned-out to be a very fundamental tool.

\section{References}

[1] CMS collaboration, R. Adolphi et al., The CMS experiment at the CERN LHC, 2008 JINST 3 S08004.

[2] D. Kotlinski et al., The control and readout systems of the CMS pixel barrel detector, Nucl. Instrum. Meth. A 565 (2006) 73.

[3] S. Das and C. Veelken, The control and readout systems of the CMS pixel detector, in preparation.

[4] PVSS made by ETM Professional Control AG (a subsidiary company of Siemens AG), Kasernenstrasse 29, 7000 Eisenstadt, Austria, http://www.pvss.com.

[5] Interface for accessing the PVSS system through SOAP XML protocol, https://twiki.cern.ch/twiki/bin/view/XdaqWiki/PSX.

[6] S. Paoletti et al., The Powering Scheme of the CMS Silicon Strip Tracker, talk at 10th Workshop on electronics for LHC and future experiments, Boston, MA, U.S.A., September 13-17 2004, CERN 2004-010, CERN-LHCC-2004-030, LHCC-G-081.

\footnotetext{
${ }^{11}$ At the moment, about once per month.
} 
[7] R. Fenner and E. Zdankiewicz, Micromachined Water Vapor Sensors: A Review of Sensing Technologies, IEEE Sens. J. 1 (2001) 309.

[8] L. Fernandez-Hernando et al., Development of a CVD diamond Beam Condition Monitor for CMS at the Large Hadron Collider, Nucl. Instrum. Meth. A 552 (2005) 183;

A. Macpherson, Beam Condition Monitoring and radiation damage concerns of the experiment, in Proceedings of the XV LHC Project Chamonix Workshop, Divonne, France, January 23-27 2006, pg. 198 ;

D. Chong et al., Validation of synthetic diamond for a Beam Condition Monitor for the Compact Muon Solenoid Experiment, IEEE Trans. Nucl. Sci. 54 (2007) 182.

[9] H. Berger, Automating with STEP 7 in STL and SCL: programmable controllers SIMATIC S7-300/400, fourth edition, Wiley-VCH (2007).

[10] OPC server for CAEN power supplies, technical information manual, CAEN S.p.A., Viareggio (LU), Italy, available from http://www.caen.it.

[11] PVSS II Native driver for S7-300/S7-400, ETM Professional Control AG, Eisenstadt, Austria, available at http://ab-project-unicos.web.cern.ch/ab-project-unicos/S7-Unicos/docs/NativeDriver_S7.pdf.

[12] O. Holme, M. Gonzalez Berges, P. Golonka and S. Schmeling, The JCOP framework, talk at ICALEPCS 2005, Geneva, Switzerland, October 10-14 2005, see also http://itcobe.web.cern.ch/itcobe/Projects/Framework/welcome.html.

[13] F. Calheiros, P. Golonka and F. Varela, Automating the Configuration of the Control Systems of the LHC Experiments, talk at ICALEPCS 2007, Knoxville, Tennessee, October 15-19 2007.

[14] C. Gaspar and B. Franek, Tools for the automation of large distributed control systems, IEEE Trans. Nucl. Sci. 53 (2006) 974.

[15] P. Cederqvist et al., Version Management with CVS (1993), available from http://www.cvshome.org/docs/manual/. 